\title{
LA CRISIS FINANCIERA ESTADOUNIDENSE: PRINCIPALES EFECTOS MACROECONÓMICOS EN LA ECONOMÍA COSTARRICENSE EN 2008 Y 2009
}

\author{
HenRy Mora Jiménez \\ Facultad de Ciencias Sociales, Universidad Nacional, Costa Rica \\ hmoraj@una.ac.cr \\ Roxana Morales Ramos \\ Escuela de Economía, Universidad Nacional, Costa Rica \\ rmorale@una.ac.cr
}

\section{RESUMEN}

La economía costarricense es una economía pequeña y muy abierta al exterior, por ello, lo que ocurre en el resto del mundo le afecta en menor o mayor grado. Cuando estalló la crisis en los Estados Unidos, sus repercusiones se hicieron notar rápidamente en el mundo. En un principio el principal efecto sobre Costa Rica se vio reflejado en la inflación, sin embargo, posteriormente los efectos saltaron al sector real de la economía: la producción y al empleo. Aquí se analizan los principales efectos macroeconómicos que tuvo la crisis económica mundial en la economía del país durante los años 2008 y 2009; entre ellos, la inflación, la reducción en la actividad económica de los sectores construcción, industria, comercio y hoteles, principalmente; la caída en el turismo, las remesas, la Inversión Extranjera Directa (IED), las exportaciones e importaciones y, por último, la pérdida de empleos.

PALABRAS CLAVES: COSTA RICA, CRISIS ECONÓMICA, IMPACTOS MACROECONÓMICOS

\section{ABSTRACT}

Costa Rica's economy is a very small, open economy; therefore, what happens in the rest of the world affects us to some degree. When the crisis in the United States exploded, its impact slowly became evident around the world. At first, the main effect on our country was reflected in inflation; however, overtime the effects moved to the real economic sector, namely, production and employment. This article examines the main macroeconomic effects the global economic crisis has had on our economy. They include inflation, the decline in the economic activity in mainly the sectors regarding construction, industry, commerce and hotels, the fall in tourism, remittances, Foreign Direct Investment, exports and imports, and finally, the reduction in employment.

KEYWORDS: COSTA RICA, ECONOMIC CRISIS, MACROECONOMIC IMPACTS

\section{INTRODUCCIÓN}

La economía costarricense es una economía pequeña y muy abierta al exterior, por tanto, también es muy susceptible a los cambios que se den en el entorno internacional.

La crisis mundial 2008-2009 tuvo su inicio en el sector financiero de la economía estadounidense, principal economía del planeta. Rápidamente sus efectos se trasladaron al sector real y también al resto del mundo. 
Costa Rica no salió librado de sus efectos, y desde los primeros meses del 2008 se empezaron a mostrar los síntomas de contagio: empezó a desacelerarse el ritmo de crecimiento de la producción, se incrementó la inflación, la entrada de remesas comenzó a caer al igual que la inversión extranjera y las exportaciones, la entrada de turistas se redujo, el consumo mermó y el desempleo se incrementó notablemente.

El objetivo del presente trabajo es realizar un análisis detallado de los principales impactos macroeconómicos que tuvo la crisis financiera internacional sobre la economía costarricense durante los años 2008 y 2009. Es importante aclarar, que por motivos de espacio y de enfoque, no se incluye un estudio de las principales políticas públicas implementadas, sino un análisis a partir de los principales indicadores económicos disponibles. Además, aunque el artículo se centra en los efectos de la crisis, ello no sugiere que la crisis vivida en el ámbito interno solamente respondió a un fenómeno externo; esto porque el modelo de desarrollo o estrategia de crecimiento impulsado por el país en las últimas décadas también es responsable de que nuestra economía haya quedado particularmente expuesta a lo sucedido en la economía mundial.

En el primer apartado se analiza la producción general y sectorial; en el segundo la inflación y el costo de la vida. En el tercero se estudia la balanza de pagos y dentro de ella las exportaciones, las importaciones, las remesas y la Inversión Extranjera Directa (IED). En el cuarto apartado se presentan las implicaciones de dicha crisis sobre las finanzas públicas; en el quinto se realiza un breve análisis sobre el impacto en el sector turismo; en el sexto se lleva a cabo un análisis sobre el empleo general y por sectores y; finalmente, en el último apartado se exponen algunas conclusiones generales del artículo.

\section{Impacto sobre la producción}

El impacto de la crisis económica sobre el nivel de producción empezó a manifestarse desde comienzos del año 2008 cuando el Índice Mensual de Actividad Económica (IMAE) inició su tendencia de desaceleración (ver Gráfico 1.1). Durante todo el año 2008, la desaceleración fue muy evidente y pronunciada, tanto que desde el mes de setiembre de dicho año y hasta el mes de agosto del 2009, la variación interanual del IMAE fue negativa (-0,49\% al mes de agosto del 2009), acumulándose 12 meses consecutivos de decrecimiento en la producción, según este indicador. Después de setiembre del 2009 el indicador empezó a mostrar mejoras, es decir, comenzó a acumular tasas de crecimiento positivas, sin embargo, no fue hasta el mes de diciembre de dicho año que se lograron alcanzar los niveles de producción que se mantenían en febrero del 2008 (nivel más alto alcanzado antes del inicio de la crisis).

\section{GRÁFICO 1.1}

COSTA RICA: ÍNDICE MENSUAL DE ACTIVIDAD ECONÓMICA GENERAL (ENERO DEL 2006-DICIEMBRE DEL 2009)

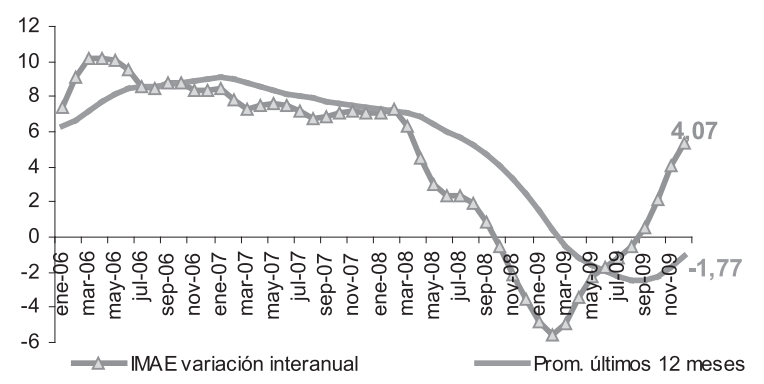

Fuente: BCCR.
1. El IMAE es un índice de quántum tipo Laspeyres y mide la evolución de la actividad económica, aproximando el comportamiento mensual del valor agregado de las diferentes industrias incluidas en el cálculo del Producto Interno Bruto. En la mayoría de los casos, asume una razón insumo-producto fijo para cada industria y refleja básicamente las variaciones reales que se dan en la producción 
Los sectores productivos más afectados fueron el sector construcción, industria manufacturera, comercio, hoteles y agropecuario; aunque hay que señalar que este último también fue seriamente impactado por los problemas climatológicos en el país.2

Como se puede apreciar en el Gráfico 1,2, el "sector construcción" mostró un acelerado ritmo de crecimiento en el bienio 2006-2007, alcanzando un máximo de 27,3\% (variación interanual) ${ }^{3}$ en mayo del 2007. Los grandes proyectos hoteleros en las zonas costeras de nuestro país, principalmente en la costa Pacífica; los grandes condominios; los centros comerciales y la gran cantidad de viviendas en todo el territorio nacional constituyen una clara evidencia de la gran actividad que presentó el sector en dicho período.

GRÁFICO 1.2

COSTA RICA: IMAE SECTOR CONSTRUCCIÓN (ENERO DEL 2006-DICIEMBRE DEL 2009)

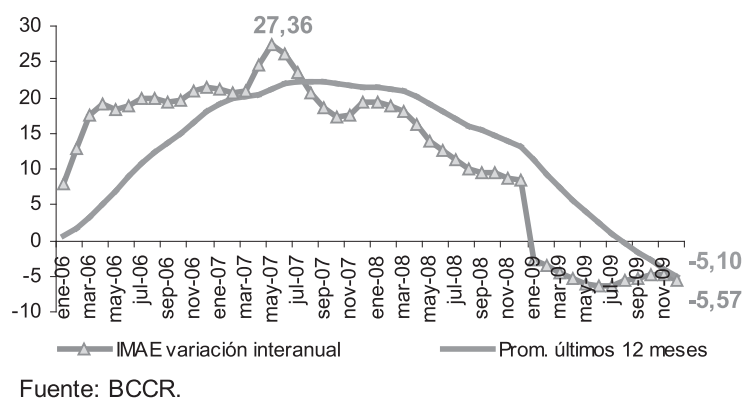

Existen varios factores que impulsaron este gran "boom de la construcción", entre ellos, la bonanza económica que se vivía en ese momento, las bajas tasas de interés (llegando a un mínimo de 4,25\% en abril del 2008), la accesibilidad al crédito tanto nacional como internacional y la cre-

2. En este artículo solo se analizan los sectores más impactados que mostraron tasas de crecimiento negativas entre octubre del 2008 y diciembre del 2009. El resto de los sectores -en su mayoría de servicios- aunque tuvieron un ritmo de crecimiento inferior al de períodos anteriores, estos no presentaron serias complicaciones que ameriten incluirlos en el análisis.

3. La variación interanual se calcula comparando el IMAE de un mes de un Año X con el IMAE del mismo mes del año anterior ciente captación de ahorro externo por parte de este sector.

No obstante, a principios del 2008 el panorama que impulsó el fuerte auge se complicó. Las tasas de interés se incrementaron en el ámbito nacional, la disponibilidad de crédito nacional e internacional se redujo, las condiciones crediticias se endurecieron en todo el mundo -incluyendo a nuestro país - y, la canalización de la IED hacia este sector se redujo, pasando de $\$ 631$ millones en el 2007 a \$432 millones en el 2008, según datos de la Memoria Anual Banco Central de Costa Rica (BCCR, 2008).

Estos hechos provocaron una desaceleración en el ritmo de crecimiento de este sector durante todo el 2008 y un decrecimiento desde el mes de enero del 2009. En el mes de diciembre, el sector decreció un 5,57\% con respecto a diciembre del 2008 (ver Gráfico 1.2); acumulando así 12 meses de decrecimiento en su nivel de actividad.

Esta menor actividad, además, provocó una gran pérdida de empleos. Según los últimos datos disponibles publicados por la Caja Costarricense de Seguro Social (CCSS), entre octubre del 2008 (mes en que inició el decrecimiento del IMAE general) y diciembre del 2009 se perdieron 24124 empleos formales en el sector construcción, evidenciándose así el efecto de la crisis.

\section{GRAFICO 1.3}

\section{COSTA RICA: IMAE SECTOR INDUSTRIAL (ENERO DEL 2006-DICIEMBRE DEL 2009)}

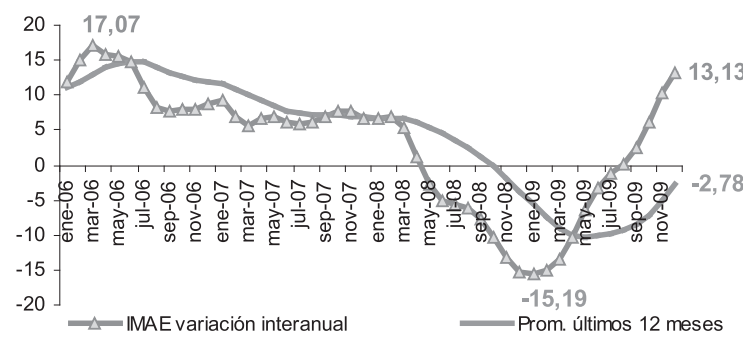

Fuente: BCCR 
La industria manufacturera o sector industrial es otro sector que también se vio fuertemente impactado por la crisis. Desde el mes de mayo del 2008 y hasta agosto del 2009 mostró tasas de crecimiento negativas, llegando a un mínimo de-15,19\% en enero del 2009 (ver Gráfico 1.3). A partir de setiembre, el nivel de actividad comenzó a mejorar, llegando a una tasa de crecimiento de 13,13\% en el mes de diciembre. De esta forma, este sector, el más perjudicado por la crisis en cuanto a nivel de producción, se convierte en el primero en salir de la recesión.

Como complemento, cabe mencionar que durante el 2009, la demanda de nuestros bienes industriales -por parte del resto del mundo- se redujo en $8,7 \%$ con respecto al 2008. Los textiles $(-26 \%)$, circuitos integrados (-18\%), alcohol etílico $(-50,6 \%)$ y aceite de palma $(-26,9 \%)$ son algunos de los productos industriales de mayor peso que redujeron sus exportaciones. Como consecuencia de ello, de octubre del 2008 a diciembre del 2009, se perdieron 8330 puestos de trabajo formales en el sector industrial.

GRÁFICO 1.4

COSTA RICA: IMAE SECTOR COMERCIO

(ENERO DEL 2006-DICIEMBRE DEL 2009)

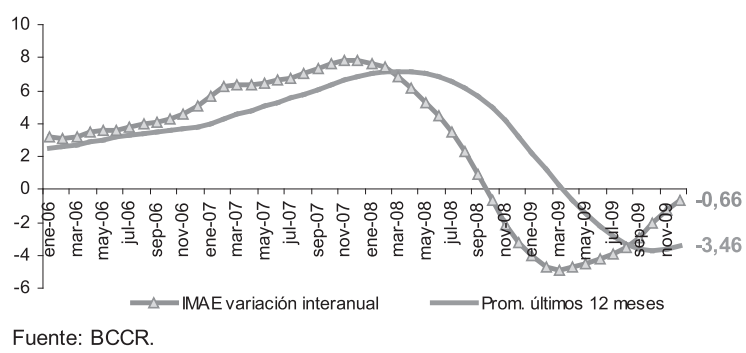

En relación con el "sector comercio", durante el 2007 su nivel de actividad fue bastante elevada con respecto al promedio histórico (ver Gráfico 1.4), impulsado en gran parte por la bonanza económica, por las bajas tasas de interés y la alta disponibilidad de crédito, la disposición de los comercios de entregar bienes duraderos sin prima y sin fiadores, y por la apreciación del colón (reduc- ción del tipo de cambio del colón con respecto al dólar) entre octubre del 2007 y mayo del 2008.

Sin embargo, este sector corrió la misma suerte que los anteriores sectores; al incrementarse las tasas de interés, el tipo de cambio, la inflación y al endurecerse las condiciones crediticias, el sector fue reduciendo su actividad y desde octubre del 2008, la tasa de crecimiento fue negativa, alcanzando un mínimo de -4,92\% en marzo del 2009 y mostrando menores caídas en los siguientes meses. En el mes de diciembre del 2009 la tasa de crecimiento fue de $-0,66 \%$.

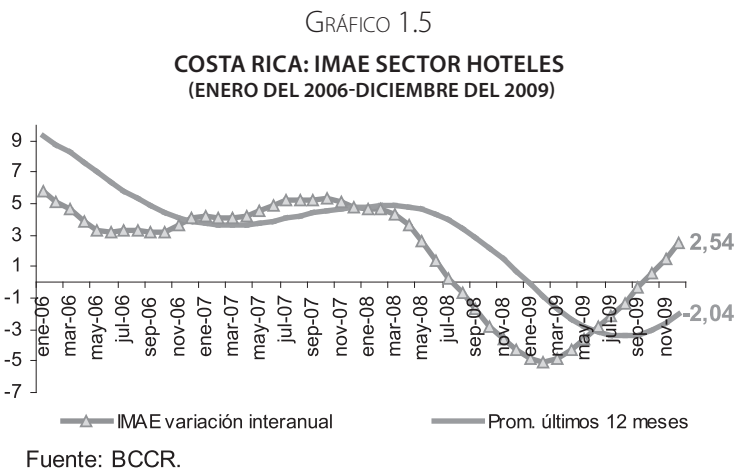

Otro sector perjudicado fue es el "sector hoteles". Dicho sector desde el mes de agosto del 2008 y hasta el mes de setiembre del 2009 mostró una fuerte contracción en su actividad. Alcanzó un mínimo de -5,04\% en febrero del 2009 y tasas de crecimiento positivas a partir de octubre del mismo año. En el mes de diciembre alcanzó una tasa de crecimiento de 2,54\% con respecto a diciembre del 2008 (ver Gráfico 1.5).

Entre las causas de dicha contracción están la menor entrada de turistas al país, la elevada inflación durante el 2008 -particularmente en combustibles- que redujo los paseos de las familias costarricenses, el incremento en las tasas de interés, la menor disponibilidad de crédito, el incremento en el tipo de cambio que encareció las tarifas y las expectativas poco favorables sobre la economía nacional y mundial. 
GRAFICO 1.6

COSTA RICA: IMAE SECTOR AGROPECUARIO

(ENERO DEL 2006-DICIEMBRE DEL 2009)

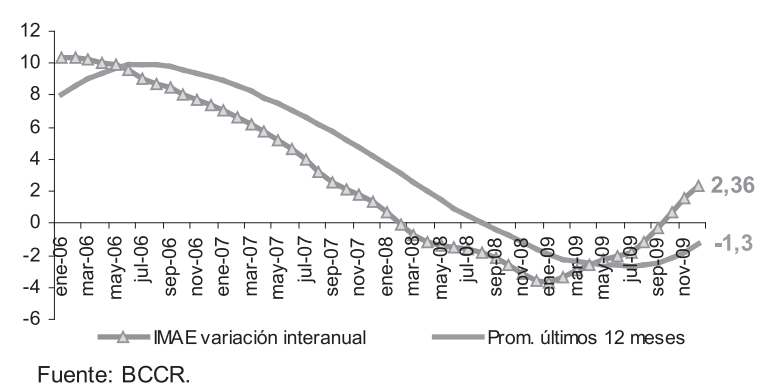

Por último, el "sector agropecuario" también se vio perjudicado por la crisis, no obstante, los mayores impactos negativos fueron producto de condiciones climatológicas adversas y de enfermedades que afectaron los cultivos de banano, café, melón, entre otros.

Desde marzo del 2008 este sector mostró contracciones en su actividad, llegando a un mínimo de -3,66\% en enero del 2009 (ver Gráfico 1.6). A partir de dicho mes y hasta setiembre, se continuaron mostrando tasas de crecimiento negativas, aunque cada mes en menor magnitud. A partir de octubre mejoró la producción, alcanzando en diciembre una tasa de crecimiento de $2,36 \%$.

Entre las causas no naturales que explican el decrecimiento presentado en este sector se puede mencionar la menor demanda externa, el encarecimiento de los fertilizantes y el costo del transporte, el incremento en las tasas de interés, la escasa disponibilidad de crédito; y en definitiva, el abandono que ha sufrido durante años dicho sector por parte del Gobierno.

\section{Inflación y costo de la vida}

Cuando estalló la crisis financiera en Estados Unidos, el primer efecto mundial que se mostró fue sobre el precio del petróleo y el de algunas materias primas.
Al dejar de ser rentable invertir en el mercado financiero, grandes inversionistas recurrieron al mercado del petróleo y al de algunas materias primas para colocar su capital; esta situación generó un alza especulativa en el precio del petróleo y sus derivados, el maíz, la soya, el trigo, el arroz, entre otros productos.

El acelerado incremento en el precio del petróleo, hasta llegar a \$133 93US por barril en junio del 2008, provocó una ola inflacionaria, donde muchos precios de bienes y servicios en el ámbito mundial se vieron afectados. A partir del mes de julio del mismo año, el precio del petróleo comenzó a bajar y con ello los diferentes precios se empezaron a estabilizar.

En Costa Rica, la subida de precios del petróleo y de los alimentos impactó significativamente.

El precio del petróleo se encareció fuertemente afectando el precio final de los combustibles -en mayor medida el precio del diésel-; los pasajes de autobús, las tarifas de taxis y el costo del boleto aéreo. Según datos de la Refinería Costarricense de Petróleo (RECOPE), el mayor nivel de precios en los combustibles se registró en el mes de octubre del 2008, cuando el precio del diésel, la gasolina Súper y la Regular se incrementaron en promedio un 36\% en relación con los precios vigentes en octubre del 2007 (ver Tabla 1).

TABLA 1

\section{COSTA RICA: PRECIO FINAL DE LOS COMBUSTIBLES}

\begin{tabular}{|llll}
\hline & \multicolumn{3}{l}{$\begin{array}{l}\text { Precio de consumo final. } \\
\text { Colones por litro }\end{array}$} \\
\cline { 2 - 4 } & SÚPER & PLUS & DIÉSEL \\
\hline $\begin{array}{l}\text { Octubre } \\
\text { del 2007 }\end{array}$ & 543 & 527 & 462 \\
$\begin{array}{l}\text { Octubre } \\
\text { del 2008 }\end{array}$ & 722 & 708 & 650 \\
Variación \% & $32,97 \%$ & $34,35 \%$ & $40,69 \%$ \\
\hline
\end{tabular}

FUENTE: RECOPE, 2008 
En relación con el incremento en los precios por grupo -durante el año 2008-, el de alimentos y bebidas no alcohólicas fue el que presentó la mayor tasa de inflación (22,8\%). Dicho incremento fue, además, mayor en 8,9 puntos porcentuales (pp.) a la inflación promedio de la economía - medida por el Índice de Precios al Consumidor (IPC) ${ }^{4}$-, la cual cerró en un 13,9\% en el mes de diciembre. Entre los alimentos que más incrementaron su precio están la papa (219\%), la yuca (104\%), el arroz (43\%), el aceite (42\%), las papas tostadas (42\%), la mantequilla (41\%), la harina de trigo (36\%) y las pastas (36\%), solo por mencionar algunos productos.

Esta situación también se ve reflejada al analizar la variación interanual del Costo de la Canasta Básica Alimentaria (CCBA) ${ }^{5}$. En el Gráfico 2.1 se observa cómo desde octubre del 2007, el costo de los 45 alimentos que componen la CBA se incrementó a un ritmo mucho mayor que el IPC.

\section{GRÁFICO 2.1}

COSTA RICA: VARIACIÓN INTERANUAL DEL IPC Y DEL COSTO DE LA CANATA BÁSICA ALIMENTARIA (ENERO DEL 2006-DICIEMBRE DEL 2009)

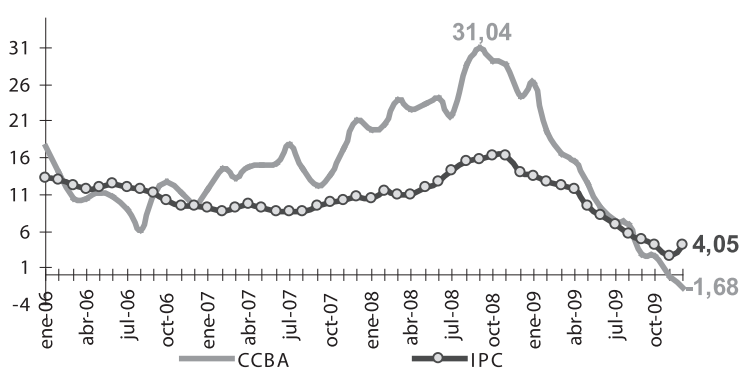

Fuente: INEC

4. El Índice de Precios al Consumidor es el indicador utilizado para medir la inflación en nuestro país y considera una canasta de 292 bienes y servicios.

5. La CBA es un conjunto de alimentos seleccionados de acuerdo con su aporte calórico y con la frecuencia de su consumo en los hogares, expresados en cantidades (gramos) que permiten satisfacer, por lo menos, las necesidades de calorías de un individuo promedio de una población de referencia. Uno de los principales usos de la CBA es para la medición de la pobreza mediante la aplicación del método de Línea de Pobreza. En Costa Rica, la CBA está conformada por 44 alimentos para la zona urbana, 37 para la zona rural y 45 a escala nacional.
En el mes de septiembre del 2008, el costo de la CBA presentó el mayor incremento con respecto al mismo mes del año anterior, creció prácticamente al doble de la inflación (31,04\% y 15,77\%, respectivamente). Esta situación reitera que el mayor efecto inflacionario en la economía durante el 2008 lo presentó el incremento en el costo de los alimentos y por lo tanto, fueron las familias de menores ingresos (ubicadas en pobreza extrema o en el primer quintil de la población) las más perjudicadas, al ser estas las que dedican el mayor porcentaje de su ingreso al consumo de alimentos.

El segundo grupo de mayor inflación durante el 2008 fue el de Comidas y bebidas fuera del hogar (17,25\%), el tercero, el de Bebidas alcohólicas y cigarrillos $(15,62 \%)$ y, el cuarto, el de Alquiler y servicios de la vivienda $(15,4 \%)$.

A partir del último trimestre del 2008 se empezó a notar una reducción en el precio de los alimentos y del petróleo, lo que permitió, junto con la menor demanda interna, que durante el 2009 la inflación acumulada al mes de diciembre alcanzara tan solo un 4,05\%, porcentaje mucho menor al 13,9\% acumulado al mes de diciembre del 2008; lo que evidencia que los efectos de la crisis internacional en el 2009 no se mostraron sobre la inflación o sector monetario, sino más bien sobre el sector real de la economía, es decir, sobre la producción, el empleo, los ingresos y el consumo.

\section{Balanza de Pagos}

La Balanza de Pagos (BP) se define como "el registro contable de las transacciones comerciales, de servicios y de movimiento de capitales llevadas a cabo por los residentes de un país con el resto del mundo, durante un período de tiempo determinado" (Manual del La Balanza de Pagos, 1977, p.6). En Costa Rica, la crisis internacional generó importantes desequilibrios en dicha balanza. Se redujeron las exportaciones, la entrada 
de divisas por concepto de turismo, las remesas y la inversión extranjera; por el contrario, las importaciones se dispararon fuertemente durante el 2008 y mermó hacia finales del 2009.

\section{Exportaciones}

Como consecuencia de la menor demanda de nuestros productos por parte de la economía estadounidense y del resto del mundo, las exportaciones de nuestro país durante el 2008 presentaron un bajo dinamismo y desde el mes de diciembre de dicho año y hasta diciembre del 2009, estas se redujeron en comparación con las exportaciones acumuladas a los mismos meses del año anterior.

Durante el 2009, las exportaciones totales se redujeron un 9\% con respecto a las exportaciones totales del año 2008. Las exportaciones hacia Estados Unidos -principal socio comercial de Costa Rica- se redujeron un 12,7\% en el mismo período; las exportaciones hacia Asia, un -5,4\%, hacia Centroamérica, un $-8,6 \%$ y hacia Europa, un $-1,7 \%$.

Los productos de mayor peso dentro del total de exportaciones, que fueron mayormente afectados son los circuitos integrados y microestructuras eléctricas, partes para computadoras, textiles, implantes de uso médico, alcohol etílico, café, banano, entre otros.

\section{Importaciones}

Por su parte, las importaciones durante el año 2008 se incrementaron fuertemente; esto como consecuencia del mayor precio de los alimentos, materias primas, materiales para la construcción, combustibles y fertilizantes; y también como consecuencia de las bajas tasas de interés en el mercado nacional, la alta disponibilidad de crédito y las constantes fluctuaciones del tipo de cambio que, por un lado, estimulaban el consumo de bienes importados en períodos de apreciación de la moneda nacional, y por otro, encarecían el total de importaciones en períodos de devaluación.

Desde el último trimestre del 2008, la tendencia de crecientes importaciones se revirtió. La crisis afectó el bolsillo de los costarricenses y el nivel de actividad económica de nuestro país se contrajo. La reducción en el precio del petróleo y su menor consumo permitió reducir las importaciones por este concepto. La reducción en el precio de los alimentos y materias primas permitió una menor salida de divisas; y la importación de bienes de consumo, tanto "duraderos" como "no duraderos", también se contrajo.

\section{Remesas familiares}

La entrada de remesas al país también se vio levemente afectada por la crisis, particularmente a partir del tercer trimestre del 2008, cuando se redujo en un 12,04\% en comparación con el tercer trimestre del 2007. En el cuarto trimestre bajó en un 5,66\% y como promedio anual disminuyó en un 1,97\%. Pasó de \$595,6 millones en el 2007 a $\$ 583,9$ millones en el 2008.

Según los datos disponibles al momento de redactar este artículo, en los primeros nueve meses del 2009 las remesas se redujeron en un 12,22\%, con respecto al mismo período del 2008. Pasaron de $\$ 430,11$ millones a $\$ 377,57$ millones.

Es importante mencionar que alrededor del 71\% de las remesas que recibe Costa Rica provienen de los Estados Unidos (Banco Mundial, 2008) y, que cerca del 23\% de los hombres costarricenses que laboran en ese país, lo hacen en el "sector construcción" (Banco Mundial, 2008)-sector más golpeado por la crisis-; por ello, era de esperar que el envío de remesas hacia nuestro país se contrajera. 


\section{Inversión Extranjera Directa}

En el año 2008, la Inversión Extranjera Directa (IED) en nuestro país se elevó significativamente, pasando de \$1896 millones en el 2007 a \$2021 millones en el 2008. Los sectores que más ahorro externo captaron fueron el "sector industrial" y el "sector agrícola".

El "sector industrial" contó con el 26\% del total de la IED, sobresaliendo la adquisición de una empresa costarricense por parte de una multinacional, por un monto de \$81 millones. El sector agrícola captó el 20\% del total de la IED, destacándose la compra de la totalidad de la firma costarricense Caribana (empresa exportadora de banano y piña), por parte de la transnacional Fresh del Monte Produce, por un monto de \$403 millones (\$210,740 millones) (La Nación, 10 junio del 2008).

En contraparte, los sectores que no fueron muy favorecidos en el 2008 por la IED -como consecuencia del complicado entorno internacional y particularmente de la crisis en Estados Unidosfueron el sector construcción y el sector turismo, los cuales redujeron la atracción de ahorro externo hacia dichos sectores.

En los primeros nueve meses del 2009, la IED se redujo un $38,79 \%$ con respecto a la IED de los primeros nueves meses del 2008; es decir, paso de $\$ 1616,44$ millones a $\$ 989,42$ millones.

Para finales del 2009, el BCCR, en su programación macroeconómica, estimó que el total de la IED hacia Costa Rica podría ubicarse en \$1300 millones durante el 2009; lo que refleja una reducción de $\$ 721$ millones con respecto al total de la IED del 2008.

\section{Balance general}

Durante el primer semestre del 2008 el déficit de cuenta corriente ${ }^{6}$ de la BP se incrementó fuertemente (un 247\% con respecto al del primer

6. La cuenta corriente de la Balanza de Pagos registra todas las exportaciones e importaciones de bienes y servicios realizadas por el país en un periodo determinado. semestre del 2007) como consecuencia del gran aumento en las importaciones y el bajo dinamismo de las exportaciones. Esta situación no fue valorada como alarmante por las autoridades económicas, debido a que durante ese mismo semestre la mayor entrada de divisas por concepto de turismo, remesas e IED compensaba holgadamente dicho déficit; no obstante, como se expuso antes, la crisis mundial afectó estos últimos tres indicadores y, durante el segundo semestre del 2008, la cuenta de capital dejó de financiar el déficit en cuenta corriente.

En los primeros nueve meses del 2009, una caída más pronunciada en las importaciones que en las exportaciones permitió que se registrara un déficit de cuenta corriente por un monto de $\$ 290,9$ millones. Monto muy inferior al registrado en los primeros nueve meses del 2008 ( $\$-2184,4$ millones).

Con respecto a la cuenta de capitales, en los primeros nueve meses del 2009, la entrada de capitales se redujo un 65,9\% en relación con la de los primeros nueve meses del 2008. Pasó de acumular \$1769,24 millones en el 2008 a \$608,81 millones en el 2009. A pesar de la fuerte caída en la cuenta de capitales, ello no afectó la BP debido a que la cuenta corriente no requirió gran financiamiento en comparación con el 2008.

Durante el 2009, se presentó una mayor caída en las importaciones que en las exportaciones, como producto de la reducción en el precio de los combustibles, alimentos y materias primas y como consecuencia de la menor demanda de bienes de inversión y bienes de consumo (tanto bienes duraderos como no duraderos). Por ello, el BCCR calcula que el déficit en la cuenta corriente del 2009 rondó el 4,9\% del PIB.

\section{Situación fiscal}

El Balance Fiscal de los años 2007, 2008 dio como resultado un superávit de $\$ 86,753$ millones y $\$ 36,364$ millones, respectivamente. Dichos 
resultados fueron consecuencia de la mayor tasa de crecimiento en la recaudación de impuestos por conceptos de renta, aduanas y ventas y; de la menor tasa de crecimiento del gasto en relación con los ingresos.

A pesar de que el balance general del año 2008 presentó un superávit financiero, este fue ¿50389 millones menor con respecto al del 2007. Esta reducción se debe a que en los últimos meses del año la crisis económica mundial empezó a golpear más fuertemente el sector real de la economía, es decir, la actividad económica se redujo y la recaudación de impuestos por conceptos de renta, ventas, aduanas, y derechos de exportación se vieron afectados. Además, para enfrentar la crisis el gobierno se vio obligado a incrementar su gasto dando como resultado el balance ya mencionado.
Por su parte, el balance del 2009 muestra de mejor manera los efectos negativos que tuvo la crisis mundial sobre las finanzas públicas de Costa Rica. La crisis, como ya se ha mencionado, afectó el nivel de la actividad económica. Las importaciones se contrajeron, afectando la recaudación en aduanas (-16,1\% con respecto a al 2008); las exportaciones redujeron, afectando la recaudación en derechos de exportación (-55,4\%); las utilidades mermaron, afectando la recaudación en renta $(-4,3 \%)$ y; la actividad comercial se redujo, afectando la recaudación en el impuesto de ventas (creció tan solo un 2,1\%). En general, los ingresos en el 2009 mostraron una reducción de -4,8\% con respecto al 2008 y los gastos totales se incrementaron un 17,7\%, arrojando un déficit financiero de 501,955 millones de colones. (ver Tabla 2).

TABLA 2

\section{COSTA RICA: VARIACIONES ANUALES EN INGRESOS Y GASTOS DEL GOBIERNO CENTRAL}

\begin{tabular}{lllll} 
& 2007 & 2008 & 2009 & PROM. \\
& VARIACIÓN $\%$ & VARIACIÓN $\%$ & VARIACIÓN \% & $2007-2009$ \\
\hline Ingresos Totales & 28,4 & 17,8 & $-4,8$ & 13,8 \\
Aduanas & 29,6 & 12 & $-16,1$ & 8,5 \\
Renta & 36,1 & 29,9 & $-1,3$ & 21,6 \\
Ventas & 25,3 & 14,5 & 2,1 & 14,0 \\
Consumo & 12,5 & 13,7 & 7,8 & 11,3 \\
Derechos de exportación & 0,6 & $-9,4$ & $-55,4$ & $-21,4$ \\
\hline Gastos totales & 17,2 & 21,2 & 17,7 & 18,7 \\
\hline
\end{tabular}

FUENTE: Elaboración propia con base en datos del BCCR. 
Para el cierre del 2009, el resultado financiero contable del Gobierno Central terminó con un déficit fiscal de alrededor de un 3,5\% del PIB, según información preliminar publicada por el Banco Central de Costa Rica.

\section{Turismo}

Según datos de la Cámara Nacional de Turismo (CANATUR) y del BCCR, el impacto de la crisis sobre este sector empezó a manifestarse hacia finales del año 2008, cuando se empezó a registrar una menor actividad turística; no obstante, como promedio anual, la entrada de turistas al país se incrementó en un 5,1\% con respecto al 2007 (se recibieron en total 2,1 millones de turistas) y el ingreso de divisas por este rubro, aumentó en un 11,25\% (se generaron ingresos superiores a los $\$ 2144$ millones).

Es claro que aunque se incrementaron, tanto la entrada de turistas como la entrada de divisas, dichos incrementos fueron inferiores a los registrados en el año anterior (14,8\% y 18,91\%, respectivamente). Además, según datos de la Memoria Anual del BCCR, y como se expuso en el apartado de Balanza de Pagos, aunque la inversión extranjera total en el país se incrementó en el 2008 con respecto al 2007, la inversión canalizada al "sector turismo" más bien se redujo, pasando de $\$ 321,3$ millones a $\$ 294$ millones.

El balance preliminar del 2009 muestra que la crisis impactó fuertemente este sector y como resultado la entrada de turistas se redujo en un $8 \%$ ( $L a$ Nación, 24 febrero del 2010) en comparación con el 2008. Ingresaron al país 167000 turistas menos que en el 2008 según cifras del Instituto Costarricense de Turismo (ICT). En el 2008 ingresaron 2,1 millones y en el 2009 1,9 millones de visitantes.

En relación con las divisas por concepto de turismo, los datos del BCCR muestran, en los primeros nueve meses del 2009, una reducción de
10,38\% con respecto al mismo período del 2008. Pasó de \$1772,6 millones a \$1588,6 millones.

\section{Empleo}

En Costa Rica, las estadísticas oficiales sobre empleo y desempleo se obtienen a partir de la Encuesta de Hogares de Propósitos Múltiples (EHPM) que realiza el Instituto Nacional de Estadística y Censos (INEC) en el mes de julio de cada año y, cuyos resultados son dados a conocer en el mes de octubre, también de cada año. Es decir, solo una vez al año podemos tener certeza del comportamiento del empleo en el país.

Según dicha encuesta, en el 2008 la Tasa de Desempleo Abierto se incrementó levemente, pasando de 4,6\% en el 2007 a 4,9\%; no obstante, si analizamos las principales variables macroeconómicas podemos observar que el mayor impacto negativo de la crisis sobre nuestro país se empezó a mostrar en el mes de octubre del 2008, cuando la variación interanual del IMAE empezó a acumular resultados negativos. Es así como el resultado de la tasa de desempleo del 2009 mostró un fuerte incremento ubicándose en un 7,8\% de la población económicamente activa. Además, la tasa de subutilización de la mano de obra ${ }^{7}$ pasó de un 11\% en el 2008 a un 15\% en el 2009. Los resultados de la Encuesta de Hogares del 2009 también mostraron como el subempleo visible, es decir, las personas que trabajan menos de la jornada laboral establecida en el código de trabajo (48 horas) se incrementó, pasando de 3,8\% en el 2008 a 4,9\% en el 2009.

Por su parte, el único indicador que puede dar algunas señales sobre el comportamiento del empleo a corto plazo -aunque solamente del

7. La Tasa de Subutilización es un Indicador resumen acerca del problema de la subutilización de la mano de obra. Se define como la suma de las tasas de desempleo abierto, de subempleo visible y de subempleo invisible. 
empleo formal- es la cantidad de trabajadores reportados a la CCSS. Desde el mes de octubre del 2008 y hasta el mes de diciembre del 2009, hubo incremento neto solamente de 1.201 puestos de trabajo. Si analizamos por sectores, en este mismo período hubo una perdida de 24121 empleos en el "Sector Construcción" (sector privado), 8330 en la "Industria Manufacturera", 3067 en "Actividad Desconocida" y 1132 en el "Intermediación Financiera", entre otros, que suman un total de 37,491 empleos perdidos. Por su parte, la cantidad de nuevos empleos se incrementó en 38692 (ver Figura 6.1). Es importante señalar que aunque al mes de diciembre se incrementaron los puestos de trabajo en 1201, este dato resulta muy insuficiente para captar la nueva fuerza laboral que año con año se incorpora al mercado laboral, por lo que el desempleo al cierre del 2009 continuó siendo un gran problema para la economía y su reducción un reto para las autoridades económicas del país.

FIGURA 6.1

COSTA RICA: CANTIDAD DE EMPLEADOS PERDIDOS

DE OCTUBRE DEL 2008 A DICIEMBRE DEL 2009 POR RAMA DE ACTIVIDAD ECONÓMICA

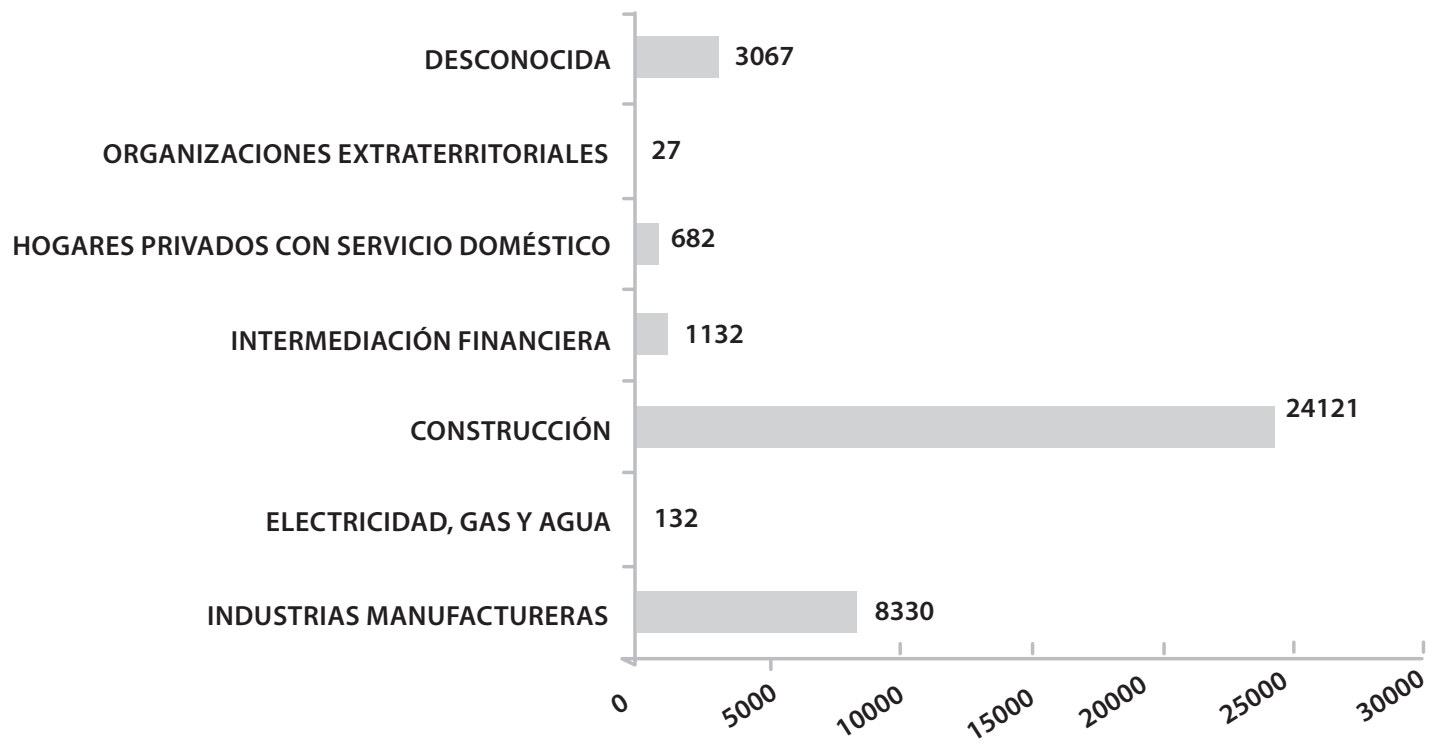

FUENTE: Elaboración propia con base de datos de la CCSS.

NotA: Basado en el reporte mensual de la CCSS sobre Seguros de Salud por Rama de Actividad Económica. Total de empleos perdidos (de octubre del 2008 a diciembre del 2009) 37 491. Se generaron 30692 nuevos empleos, para un saldo de 1201 puestos de trabajo.

Adicionalmente, podemos observar el impacto de la crisis sobre el empleo, mediante una recopilación de datos en los principales medios de comunicación nacional.
En la Tabla 3 se mencionan algunos de los despidos masivos realizados por empresas reconocidas en los últimos meses del 2008 y primeros meses del 2009. 
Tabla 3

\section{COSTA RICA: ALGUNOS DESPIDOS EN EMPRESAS DEL SECTOR PRIVADO (2008- 2009)}

\begin{tabular}{|c|c|}
\hline EMPRESA & EMPLEOS PERDIDOS \\
\hline Importadora Monge & $\begin{array}{l}\text { Grupo M durante el } 2008 \text { hizo un recorte del } 14 \% \text { de su planilla en C.R. } \\
\text { Despidió un total de } 475 \text { empleados. Esta empresa tiene aproximadamente } \\
2800 \text { empleados en C.R. }\end{array}$ \\
\hline Sábila Industrial & $\begin{array}{l}\text { Ubicada en Liberia, bajo el régimen de Zona Franca, despide en el mes de } \\
\text { enero a } 160 \text { empleados. }\end{array}$ \\
\hline Domino's Pizza & 130, la mayoría mujeres y muchas de ellas jefas de hogar. \\
\hline Havells Sylvania & 200 \\
\hline Merck Sharp \& Dome & 104 \\
\hline Industrias Helvex & 25 \\
\hline Tikal, & 30 \\
\hline Hotel Marriot San José & 80 \\
\hline Hotel Marriot Los Sueños & 60 \\
\hline Mabe (antigua Atlas), & 300, desde el mes de noviembre del 2008 hasta la fecha. \\
\hline Vidriera Centroamericana & Número sin precisar. \\
\hline Florida Ice \& Farm & 136 en la Cervecería Costa Rica y 57 en Reserva Conchal. \\
\hline Capris & Varias decenas de trabajadores. \\
\hline Wal-Mart & 60 \\
\hline Grupo Nación & 100 \\
\hline HSBC & 24 (2\% de la planilla = 1100 trabajadores $).$ \\
\hline Scotiabank & 200 puestos (febrero del 2009). \\
\hline Bor Kar & Empresa textilera, despidió en febrero del 2009 a 180 empleados. \\
\hline Grupo Pelón de Costa Rica & $\begin{array}{l}\text { Empresa ubicada en Guanacaste y dedicada al cultivo de melón. Despidió a } \\
3000 \text { empleados debido al incremento de } 25 \% \text { en los costos de producción } \\
\text { y a la baja rentabilidad actual. (Noviembre del 2008). }\end{array}$ \\
\hline TOTAL & 5321 despidos \\
\hline
\end{tabular}

FUENTE: Datos recopilados en medios de comunicación nacional.

\section{CONCLUSIONES}

La crisis económica mundial originada en los Estados Unidos ha tenido severas repercusiones alrededor del mundo y no sabemos con certeza, cuánto tiempo más se prolongará. Sabemos que cuanto más larga y profunda sea una crisis, más difícil y lenta será la recuperación, por ello, los go- biernos han tenido que tomar una serie de medidas para evitar que los problemas ya presentados en la economía se intensifiquen y así evitar que más sectores se contagien de la crisis.

En nuestro país, como se mencionó a lo largo de este documento, la crisis empezó afectando la inflación, a causa del incremento internacional en 
los precios de los alimentos, materias primas y el petróleo; luego el efecto se sintió sobre las exportaciones, el turismo, las remesas y la IED. Esta situación provocó que la actividad económica general y de algunos sectores - particularmente de aquellos más vinculados con el mercado externo- se contrajera; que las utilidades de las empresas se redujeran, al igual que el consumo, los ingresos y la recaudación fiscal; además que los despidos laborales estuvieran la orden del día y que la incertidumbre imperara en los distintos mercados.

Durante el 2008, el principal problema que enfrentó nuestra economía fue la inflación, en el 2009 el efecto se concentró sobre el sector real, es decir, sobre la producción y el empleo. Muchas familias de bajos ingresos fueron las más golpeadas por la crisis económica, en el 2008 como producto del fuerte incremento en los precios de los alimentos, electricidad, agua y transporte público y; en el 2009, ya no por la subida de precios sino más bien por la insuficiencia de ingresos para satisfacer sus necesidades básicas. Como se mencionó en el primer apartado, entre los sectores más perjudicados por la crisis se encuentran el sector construcción, hoteles, comercio y maquila, sectores en los cuales se emplea gran cantidad de mano de obra de baja calificación y proveniente de familias pobres o relativamente pobres; por lo tanto, la pérdida de empleos en estos sectores impactó negativamente sobre el ingreso y el bienestar de dichas familias.

\section{REFERENCIAS}

Academia de Centroamérica (2008). Costa Rica 2007: crecimiento impulsado por la inversión extranjera. Editores: Luis Mesalles y Oswald Céspedes. San José, Costa Rica: Academia de Centroamérica. Disponible en: http://www.capitales.com/costarica/canales/academiaca/documentos.php?ca te $=$ Libros\%20y\%20Estudios

Banco Central de Costa Rica (2008). Memoria Anual del 2008. Publicado en la página Web del BCCR. Recuperado el 20-01-2010 de: http://www.bccr.fi.cr/flat/ bccr_flat.htm
Banco Central de Costa Rica (2008). Informes mensuales de la situación económica del país. Recuperado el 20-012010 de: http://www.bccr.fi.cr/flat/bccr_flat.htm

Banco Central de Costa Rica (2009). Informes mensuales de la situación económica del país. Recuperado el 20-012010 de: http://www.bccr.fi.cr/flat/bccr_flat.htm

Banco Mundial (2008). Outlook for remittance flows 2008-2010. Migration and Development. Brief, n. ${ }^{\circ}$ 8. Recuperado el 10-01-2010 de http://siteresources.worldbank.org/INTPROSPECTS/Resources/334934-1110315015165/MD_Brief8.pdf

Instituto Nacional de Estadística y Censos (2007). Encuesta de Hogares de Propósitos Múltiples. Recuperado el 2012-2009dehttp://www.inec.go.cr/botonesAccesoDir/ 04EncuestaDeHogares/01Set.htm

Instituto Nacional de Estadística y Censos (2008). Encuesta de Hogares de Propósitos Múltiples. Recuperado el 2012-2009dehttp://www.inec.go.cr/botonesAccesoDir/ 04EncuestaDeHogares/01Set.htm

Instituto Nacional de Estadística y Censos (2009). Encuesta de Hogares de Propósitos Múltiples. Recuperado el 2012-2009dehttp://www.inec.go.cr/botonesAccesoDir/ 04EncuestaDeHogares/01Set.htm

La Nación (2008). Fresh Del Monte compró bananera y piñera nacional en $\$ 400$ millones. Recuperado el 10-01-2010 de http://www.nacion.com/In ee/2008/junio/10/economia1571729.html

La Nación (2010). Llegada de turistas se redujo 8\%en 2009. Recuperado el 24-02-2010 de http://ww.nacion.com/ In_ee/2010/febrero/24/economia2278933.html

Promotora de Comercio Exterior (2008). Informes mensuales sobre exportaciones. Recuperado el 20-12-2009 de http://www.procomer.com/Espanol/Estadisticas04/mensual-04-02/est_mensual-04-02-01.htm I

Promotora de Comercio Exterior (2009). Informes mensuales sobre exportaciones. Recuperado el 20-12-2009 de http://www.procomer.com/Espanol/Estadisticas-04/mensual-04-02/est_mensual-04-02-01.html

RECIBIDO: 28-10-2009

ACEPTADO: 15-01-2009 
ÍNDICE MENSUAL DE ACTIVIDAD ECONÓMICA /N1 TENDENCIA CICLO:TASAS DE VARIACIÓN INTERANUAL /N2

$1991=100$

\begin{tabular}{|c|c|c|c|c|c|c|c|c|c|}
\hline & \multicolumn{3}{|c|}{ CON INDUSTRIA ELECTRONICA } & \multicolumn{3}{|c|}{$\begin{array}{c}\text { AGRICULTURA, } \\
\text { SILVICULTURA Y PESCA }\end{array}$} & \multicolumn{3}{|c|}{ INDUSTRIA MANUFACTURERA } \\
\hline & & $\begin{array}{c}\text { IMAE } \\
\text { variación } \\
\text { interanual }\end{array}$ & $\begin{array}{l}\text { Prom.últi- } \\
\text { mos } 12 \\
\text { meses }\end{array}$ & & $\begin{array}{c}\text { IMAE } \\
\text { variación } \\
\text { interanual }\end{array}$ & $\begin{array}{l}\text { Prom. úl- } \\
\text { timos } 12 \\
\text { meses }\end{array}$ & & $\begin{array}{c}\text { IMAE } \\
\text { variación } \\
\text { interanual }\end{array}$ & $\begin{array}{l}\text { Prom. } \\
\text { últimos } \\
12 \text { meses }\end{array}$ \\
\hline Jan-03 & 171,36 & & & 139,81 & & & 200,28 & & \\
\hline Feb-03 & 172,48 & & & 140,57 & & & 201,47 & & \\
\hline Mar-03 & 173,14 & & & 141,36 & & & 202,45 & & \\
\hline Apr-03 & 173,65 & & & 142,20 & & & 203,35 & & \\
\hline May-03 & 174,21 & & & 142,98 & & & 204,19 & & \\
\hline Jun-03 & 174,86 & & & 143,76 & & & 204,82 & & \\
\hline Jul-03 & 175,65 & & & 144,64 & & & 205,33 & & \\
\hline Aug-03 & 176,37 & & & 145,55 & & & 205,51 & & \\
\hline Sep-03 & 177,27 & & & 146,36 & & & 206,26 & & \\
\hline Oct-03 & 178,42 & & & 147,01 & & & 207,55 & & \\
\hline Nov-03 & 179,32 & & & 147,52 & & & 208,12 & & \\
\hline Dec-03 & 179,83 & & & 147,85 & & & 208,30 & & \\
\hline Jan-04 & 180,34 & 5,24 & & 148,09 & 5,93 & & 209,02 & 4,36 & \\
\hline Feb-04 & 181,23 & 5,08 & & 148,43 & 5,59 & & 210,50 & 4,48 & \\
\hline Mar-04 & 182,28 & 5,28 & & 148,91 & 5,34 & & 211,92 & 4,68 & \\
\hline Apr-04 & 182,95 & 5,36 & & 149,23 & 4,95 & & 212,70 & 4,60 & \\
\hline May-04 & 183,17 & 5,14 & & 149,26 & 4,39 & & 212,63 & 4,13 & \\
\hline Jun-04 & 183,40 & 4,88 & & 149,25 & 3,81 & & 211,97 & 3,49 & \\
\hline Jul-04 & 183,77 & 4,62 & & 149,25 & 3,19 & & 211,24 & 2,88 & \\
\hline Aug-04 & 184,06 & 4,36 & & 149,31 & 2,58 & & 210,71 & 2,53 & \\
\hline Sep-04 & 184,76 & 4,23 & & 149,65 & 2,25 & & 212,29 & 2,92 & \\
\hline Oct-04 & 185,98 & 4,24 & & 150,30 & 2,23 & & 215,66 & 3,91 & \\
\hline Nov-04 & 187,25 & 4,42 & & 151,27 & 2,54 & & 218,61 & 5,04 & \\
\hline Dec-04 & 188,46 & 4,80 & & 152,40 & 3,07 & & 221,44 & 6,31 & \\
\hline Jan-05 & 189,08 & 4,85 & 4,77 & 153,52 & 3,67 & 3,6 & 222,41 & 6,40 & 4,28 \\
\hline Feb-05 & 189,00 & 4,29 & 4,70 & 154,75 & 4,26 & 3,5 & 220,64 & 4,82 & 4,31 \\
\hline Mar-05 & 189,64 & 4,04 & 4,60 & 156,13 & 4,85 & 3,5 & 221,31 & 4,43 & 4,29 \\
\hline Apr-05 & 191,23 & 4,52 & 4,53 & 157,51 & 5,55 & 3,5 & 225,52 & 6,03 & 4,41 \\
\hline May-05 & 192,63 & 5,16 & 4,53 & 158,81 & 6,40 & 3,7 & 228,89 & 7,65 & 4,70 \\
\hline Jun-05 & 194,32 & 5,95 & 4,62 & 160,31 & 7,41 & 4,0 & 233,13 & 9,99 & 5,24 \\
\hline Jul-05 & 196,72 & 7,05 & 4,83 & 162,02 & 8,56 & 4,4 & 240,76 & 13,98 & 6,17 \\
\hline Aug-05 & 198,56 & 7,87 & 5,12 & 163,48 & 9,49 & 5,0 & 247,02 & 17,23 & 7,39 \\
\hline
\end{tabular}




\begin{tabular}{|c|c|c|c|c|c|c|c|c|}
\hline \multicolumn{3}{|c|}{ CONSTRUCCIÓN } & \multicolumn{3}{|c|}{ COMERCIO } & \multicolumn{3}{|c|}{ HOTELES } \\
\hline & $\begin{array}{c}\text { IMAE } \\
\text { variación } \\
\text { interanual }\end{array}$ & $\begin{array}{l}\text { Prom. } \\
\text { últimos } \\
12 \text { meses }\end{array}$ & & $\begin{array}{l}\text { IMAE va- } \\
\text { riación in- } \\
\text { teranual }\end{array}$ & $\begin{array}{l}\text { Prom. } \\
\text { últimos } \\
12 \text { meses }\end{array}$ & & $\begin{array}{c}\text { IMAE } \\
\text { variación } \\
\text { interanual }\end{array}$ & $\begin{array}{l}\text { Prom. } \\
\text { últimos } \\
12 \text { meses }\end{array}$ \\
\hline 186,42 & & & 145,91 & & & 268,48 & & \\
\hline 190,06 & & & 146,30 & & & 269,81 & & \\
\hline 188,92 & & & 146,66 & & & 271,30 & & \\
\hline 182,78 & & & 146,94 & & & 273,01 & & \\
\hline 176,78 & & & 147,27 & & & 275,29 & & \\
\hline 175,20 & & & 147,64 & & & 278,04 & & \\
\hline 175,35 & & & 148,02 & & & 281,25 & & \\
\hline 175,10 & & & 148,42 & & & 284,39 & & \\
\hline 176,51 & & & 148,71 & & & 287,27 & & \\
\hline 180,49 & & & 149,02 & & & 290,94 & & \\
\hline 183,65 & & & 149,44 & & & 295,09 & & \\
\hline 184,10 & & & 149,87 & & & 298,84 & & \\
\hline 185,18 & $-0,66$ & & 150,28 & 2,99 & & 302,40 & 12,63 & \\
\hline 186,72 & $-1,75$ & & 150,63 & 2,96 & & 305,61 & 13,27 & \\
\hline 186,42 & $-1,32$ & & 150,89 & 2,89 & & 307,92 & 13,50 & \\
\hline 186,49 & 2,03 & & 151,11 & 2,84 & & 310,54 & 13,75 & \\
\hline 189,53 & 7,21 & & 151,28 & 2,73 & & 314,00 & 14,06 & \\
\hline 194,26 & 10,88 & & 151,42 & 2,56 & & 316,80 & 13,94 & \\
\hline 197,40 & 12,57 & & 151,57 & 2,40 & & 319,13 & 13,47 & \\
\hline 196,82 & 12,40 & & 151,74 & 2,24 & & 321,76 & 13,14 & \\
\hline 194,74 & 10,33 & & 151,88 & 2,14 & & 324,54 & 12,97 & \\
\hline 195,60 & 8,37 & & 151,99 & 2,00 & & 327,34 & 12,51 & \\
\hline 197,83 & 7,72 & & 152,12 & 1,80 & & 330,22 & 11,91 & \\
\hline 196,55 & 6,76 & & 152,28 & 1,61 & & 333,35 & 11,55 & \\
\hline 192,67 & 4,05 & 6,60 & 152,50 & 1,48 & 2,30 & 336,60 & 11,31 & 12,95 \\
\hline 189,52 & 1,50 & 6,88 & 152,88 & 1,50 & 2,18 & 339,58 & 11,12 & 12,77 \\
\hline 185,59 & $-0,44$ & 6,95 & 153,25 & 1,57 & 2,07 & 342,38 & 11,19 & 12,58 \\
\hline 182,42 & $-2,18$ & 6,60 & 153,60 & 1,65 & 1,97 & 345,49 & 11,25 & 12,37 \\
\hline 184,22 & $-2,80$ & 5,76 & 154,13 & 1,88 & 1,90 & 348,11 & 10,86 & 12,10 \\
\hline 187,77 & $-3,34$ & 4,58 & 154,71 & 2,17 & 1,87 & 349,55 & 10,34 & 11,80 \\
\hline 191,35 & $-3,06$ & 3,28 & 155,18 & 2,38 & 1,87 & 350,44 & 9,81 & 11,50 \\
\hline 196,08 & $-0,37$ & 2,21 & 155,61 & 2,55 & 1,89 & 351,89 & 9,36 & 11,18 \\
\hline
\end{tabular}




\begin{tabular}{|c|c|c|c|c|c|c|c|c|c|}
\hline & \multicolumn{3}{|c|}{ CON INDUSTRIA ELECTRONICA } & \multicolumn{3}{|c|}{$\begin{array}{c}\text { AGRICULTURA, } \\
\text { SILVICULTURA Y PESCA }\end{array}$} & \multicolumn{3}{|c|}{ INDUSTRIA MANUFACTURERA } \\
\hline & & $\begin{array}{c}\text { IMAE } \\
\text { variación } \\
\text { interanual }\end{array}$ & $\begin{array}{l}\text { Prom.últi- } \\
\text { mos } 12 \\
\text { meses }\end{array}$ & & $\begin{array}{c}\text { IMAE } \\
\text { variación } \\
\text { interanual }\end{array}$ & $\begin{array}{l}\text { Prom. úl- } \\
\text { timos } 12 \\
\text { meses }\end{array}$ & & $\begin{array}{c}\text { IMAE } \\
\text { variación } \\
\text { interanual }\end{array}$ & $\begin{array}{l}\text { Prom. } \\
\text { últimos } \\
12 \text { meses }\end{array}$ \\
\hline Sep-05 & 199,34 & 7,89 & 5,42 & 164,71 & 10,07 & 5,7 & 247,91 & 16,78 & 8,55 \\
\hline Oct-05 & 199,94 & 7,50 & 5,70 & 165,92 & 10,39 & 6,4 & 246,86 & 14,47 & 9,43 \\
\hline Nov-05 & 201,14 & 7,42 & 5,95 & 167,03 & 10,42 & 7,0 & 248,40 & 13,63 & 10,14 \\
\hline Dec-05 & 202,46 & 7,43 & 6,16 & 168,22 & 10,38 & 7,6 & 249,52 & 12,68 & 10,67 \\
\hline Jan-06 & 203,73 & 7,75 & 6,41 & 169,67 & 10,52 & 8,2 & 249,45 & 12,16 & 11,15 \\
\hline Feb-06 & 206,00 & 8,99 & 6,80 & 171,22 & 10,64 & 8,7 & 253,87 & 15,06 & 12,01 \\
\hline Mar-06 & 208,35 & 9,86 & 7,28 & 172,56 & 10,52 & 9,2 & 259,20 & 17,12 & 13,06 \\
\hline Apr-06 & 209,81 & 9,72 & 7,72 & 173,75 & 10,31 & 9,6 & 261,17 & 15,81 & 13,88 \\
\hline May-06 & 211,45 & 9,77 & 8,10 & 174,96 & 10,17 & 9,9 & 264,11 & 15,39 & 14,52 \\
\hline Jun-06 & 212,98 & 9,60 & 8,41 & 175,96 & 9,76 & 10,1 & 267,10 & 14,57 & 14,91 \\
\hline Jul-06 & 213,96 & 8,76 & 8,55 & 176,91 & 9,19 & 10,2 & 267,46 & 11,09 & 14,67 \\
\hline Aug-06 & 214,98 & 8,27 & 8,58 & 178,05 & 8,92 & 10,1 & 267,22 & 8,18 & 13,91 \\
\hline Sep-06 & 215,97 & 8,34 & 8,62 & 179,12 & 8,75 & 10,0 & 266,95 & 7,68 & 13,15 \\
\hline Oct-06 & 216,82 & 8,45 & 8,70 & 179,99 & 8,48 & 9,8 & 266,78 & 8,07 & 12,62 \\
\hline Nov-06 & 217,98 & 8,37 & 8,78 & 180,71 & 8,19 & 9,7 & 268,55 & 8,11 & 12,16 \\
\hline Dec-06 & 219,59 & 8,46 & 8,86 & 181,41 & 7,84 & 9,4 & 271,72 & 8,90 & 11,84 \\
\hline Jan-07 & 220,96 & 8,46 & 8,92 & 182,15 & 7,36 & 9,2 & 272,59 & 9,28 & 11,60 \\
\hline Feb-07 & 221,89 & 7,71 & 8,82 & 182,86 & 6,80 & 8,9 & 271,58 & 6,97 & 10,93 \\
\hline Mar-07 & 223,42 & 7,23 & 8,60 & 183,42 & 6,30 & 8,5 & 273,48 & 5,51 & 9,96 \\
\hline Apr-07 & 225,50 & 7,48 & 8,41 & 183,81 & 5,79 & 8,1 & 277,94 & 6,42 & 9,18 \\
\hline May-07 & 227,31 & 7,50 & 8,22 & 184,04 & 5,19 & 7,7 & 281,62 & 6,63 & 8,45 \\
\hline Jun-07 & 228,55 & 7,31 & 8,03 & 184,09 & 4,62 & 7,3 & 283,36 & 6,09 & 7,74 \\
\hline Jul-07 & 229,21 & 7,13 & 7,89 & 183,93 & 3,97 & 6,8 & 283,17 & 5,87 & 7,31 \\
\hline Aug-07 & 230,06 & 7,02 & 7,79 & 183,72 & 3,18 & 6,4 & 283,45 & 6,08 & 7,13 \\
\hline Sep-07 & 231,37 & 7,13 & 7,69 & 183,66 & 2,54 & 5,9 & 285,39 & 6,91 & 7,07 \\
\hline Oct-07 & 232,74 & 7,34 & 7,59 & 183,77 & 2,10 & 5,3 & 287,85 & 7,90 & 7,05 \\
\hline Nov-07 & 233,90 & 7,30 & 7,51 & 183,93 & 1,78 & 4,8 & 289,42 & 7,77 & 7,03 \\
\hline Dec-07 & 234,76 & 6,91 & 7,38 & 183,87 & 1,36 & 4,2 & 289,57 & 6,57 & 6,83 \\
\hline Jan-08 & 235,54 & 6,60 & 7,22 & 183,52 & 0,75 & 3,7 & 289,01 & 6,02 & 6,56 \\
\hline Feb-08 & 236,23 & 6,46 & 7,12 & 183,02 & 0,09 & 3,1 & 288,24 & 6,14 & 6,49 \\
\hline Mar-08 & 236,21 & 5,72 & 6,99 & 182,50 & $-0,50$ & 2,6 & 285,85 & 4,52 & 6,41 \\
\hline Apr-08 & 235,16 & 4,28 & 6,73 & 182,11 & $-0,93$ & 2,0 & 279,88 & 0,70 & 5,93 \\
\hline May-08 & 233,82 & 2,87 & 6,34 & 181,87 & $-1,18$ & 1,5 & 272,25 & $-3,33$ & 5,10 \\
\hline Jun-08 & 233,08 & 1,99 & 5,90 & 181,73 & $-1,28$ & 1,0 & 267,20 & $-5,70$ & 4,12 \\
\hline Jul-08 & 232,89 & 1,61 & 5,44 & 181,38 & $-1,39$ & 0,5 & 265,60 & $-6,20$ & 3,11 \\
\hline
\end{tabular}




\begin{tabular}{|c|c|c|c|c|c|c|c|c|}
\hline \multicolumn{3}{|c|}{ CONSTRUCCIÓN } & \multicolumn{3}{|c|}{ COMERCIO } & \multicolumn{3}{|c|}{ HOTELES } \\
\hline & $\begin{array}{c}\text { IMAE } \\
\text { variación } \\
\text { interanual }\end{array}$ & $\begin{array}{l}\text { Prom. } \\
\text { últimos } \\
12 \text { meses }\end{array}$ & & $\begin{array}{l}\text { IMAE va- } \\
\text { riación in- } \\
\text { teranual }\end{array}$ & $\begin{array}{l}\text { Prom. } \\
\text { últimos } \\
12 \text { meses }\end{array}$ & & $\begin{array}{c}\text { IMAE } \\
\text { variación } \\
\text { interanual }\end{array}$ & $\begin{array}{l}\text { Prom. } \\
\text { últimos } \\
12 \text { meses }\end{array}$ \\
\hline 200,11 & 2,76 & 1,58 & 156,15 & 2,81 & 1,95 & 353,76 & 9,00 & 10,85 \\
\hline 201,05 & 2,79 & 1,11 & 156,73 & 3,12 & 2,04 & 354,95 & 8,43 & 10,51 \\
\hline 198,31 & 0,24 & 0,49 & 157,12 & 3,29 & 2,17 & 355,31 & 7,60 & 10,15 \\
\hline 198,61 & 1,05 & 0,02 & 157,31 & 3,30 & 2,31 & 355,41 & 6,62 & 9,74 \\
\hline 205,93 & 6,88 & 0,25 & 157,39 & 3,21 & 2,45 & 356,06 & 5,78 & 9,28 \\
\hline 214,48 & 13,17 & 1,22 & 157,58 & 3,07 & 2,58 & 357,17 & 5,18 & 8,79 \\
\hline 218,79 & 17,89 & 2,75 & 158,18 & 3,22 & 2,72 & 358,21 & 4,62 & 8,24 \\
\hline 218,17 & 19,60 & 4,57 & 159,00 & 3,51 & 2,88 & 358,98 & 3,91 & 7,63 \\
\hline 218,43 & 18,57 & 6,35 & 159,64 & 3,57 & 3,02 & 359,64 & 3,31 & 7,00 \\
\hline 223,52 & 19,04 & 8,21 & 160,29 & 3,61 & 3,14 & 360,59 & 3,16 & 6,40 \\
\hline 229,93 & 20,16 & 10,15 & 161,05 & 3,79 & 3,25 & 361,95 & 3,28 & 5,86 \\
\hline 235,50 & 20,01 & 11,85 & 161,78 & 3,97 & 3,37 & 363,46 & 3,29 & 5,35 \\
\hline 239,15 & 19,50 & 13,25 & 162,55 & 4,01 & 3,48 & 364,84 & 3,13 & 4,86 \\
\hline 241,38 & 20,06 & 14,69 & 163,34 & 4,22 & 3,57 & 366,44 & 3,24 & 4,43 \\
\hline 245,84 & 23,97 & 16,67 & 164,15 & 4,47 & 3,67 & 368,38 & 3,68 & 4,10 \\
\hline 251,62 & 26,69 & 18,80 & 165,14 & 4,98 & 3,81 & 370,08 & 4,13 & 3,89 \\
\hline 255,81 & 24,22 & 20,25 & 166,31 & 5,66 & 4,01 & 371,12 & 4,23 & 3,76 \\
\hline 258,88 & 20,70 & 20,87 & 167,41 & 6,24 & 4,28 & 371,80 & 4,09 & 3,67 \\
\hline 263,69 & 20,52 & 21,09 & 168,35 & 6,43 & 4,55 & 372,80 & 4,08 & 3,63 \\
\hline 271,55 & 24,47 & 21,50 & 169,25 & 6,45 & 4,79 & 374,14 & 4,22 & 3,65 \\
\hline 278,12 & 27,32 & 22,23 & 170,23 & 6,63 & 5,05 & 375,86 & 4,51 & 3,75 \\
\hline 281,43 & 25,91 & 22,80 & 171,24 & 6,83 & 5,31 & 378,35 & 4,93 & 3,90 \\
\hline 283,15 & 23,15 & 23,05 & 172,31 & 6,99 & 5,58 & 380,77 & 5,20 & 4,06 \\
\hline 283,73 & 20,48 & 23,08 & 173,52 & 7,26 & 5,86 & 382,27 & 5,17 & 4,22 \\
\hline 284,71 & 19,05 & 23,05 & 174,86 & 7,57 & 6,14 & 383,84 & 5,21 & 4,39 \\
\hline 285,73 & 18,37 & 22,90 & 176,27 & 7,92 & 6,45 & 385,89 & 5,31 & 4,56 \\
\hline 287,66 & 17,01 & 22,32 & 177,61 & 8,20 & 6,76 & 387,26 & 5,13 & 4,68 \\
\hline 293,05 & 16,47 & 21,47 & 178,68 & 8,20 & 7,03 & 387,94 & 4,83 & 4,74 \\
\hline 299,66 & 17,14 & 20,88 & 179,56 & 7,97 & 7,22 & 388,71 & 4,74 & 4,78 \\
\hline 304,58 & 17,65 & 20,63 & 180,18 & 7,63 & 7,34 & 389,44 & 4,74 & 4,84 \\
\hline 308,31 & 16,92 & 20,33 & 180,29 & 7,01 & 7,40 & 389,71 & 4,53 & 4,88 \\
\hline 311,04 & 14,54 & 19,50 & 179,96 & 6,33 & 7,39 & 389,35 & 4,07 & 4,86 \\
\hline 311,48 & 11,99 & 18,22 & 179,55 & 5,48 & 7,29 & 388,21 & 3,29 & 4,76 \\
\hline 310,29 & 10,25 & 16,92 & 179,12 & 4,60 & 7,10 & 386,21 & 2,08 & 4,52 \\
\hline 307,32 & 8,53 & 15,70 & 178,59 & 3,64 & 6,82 & 383,30 & 0,67 & 4,15 \\
\hline
\end{tabular}




\begin{tabular}{|c|c|c|c|c|c|c|c|c|c|}
\hline & \multicolumn{3}{|c|}{ CON INDUSTRIA ELECTRONICA } & \multicolumn{3}{|c|}{$\begin{array}{c}\text { AGRICULTURA, } \\
\text { SILVICULTURA Y PESCA }\end{array}$} & \multicolumn{3}{|c|}{ INDUSTRIA MANUFACTURERA } \\
\hline & & $\begin{array}{c}\text { IMAE } \\
\text { variación } \\
\text { interanual }\end{array}$ & $\begin{array}{l}\text { Prom.últi- } \\
\text { mos } 12 \\
\text { meses }\end{array}$ & & $\begin{array}{c}\text { IMAE } \\
\text { variación } \\
\text { interanual }\end{array}$ & $\begin{array}{l}\text { Prom. úl- } \\
\text { timos } 12 \\
\text { meses }\end{array}$ & & $\begin{array}{c}\text { IMAE } \\
\text { variación } \\
\text { interanual }\end{array}$ & $\begin{array}{l}\text { Prom. } \\
\text { últimos } \\
12 \text { meses }\end{array}$ \\
\hline Aug-08 & 232,44 & 1,03 & 4,94 & 180,69 & $-1,65$ & 0,1 & 264,02 & $-6,86$ & 2,04 \\
\hline Sep-08 & 231,35 & $-0,00$ & 4,34 & 179,84 & $-2,08$ & $-0,2$ & 260,41 & $-8,75$ & 0,73 \\
\hline Oct-08 & 229,69 & $-1,31$ & 3,62 & 178,89 & $-2,66$ & $-0,6$ & 255,18 & $-11,35$ & $-0,87$ \\
\hline Nov-08 & 227,66 & $-2,67$ & 2,79 & 177,88 & $-3,29$ & $-1,1$ & 248,68 & $-14,07$ & $-2,69$ \\
\hline Dec-08 & 226,09 & $-3,70$ & 1,91 & 176,94 & $-3,77$ & $-1,5$ & 244,02 & $-15,73$ & $-4,55$ \\
\hline Jan-09 & 225,51 & $-4,26$ & 1,00 & 176,23 & $-3,98$ & $-1,9$ & 243,64 & $-15,70$ & $-6,36$ \\
\hline Feb-09 & 225,59 & $-4,50$ & 0,09 & 175,91 & $-3,88$ & $-2,2$ & 245,16 & $-14,95$ & $-8,12$ \\
\hline Mar-09 & 226,12 & $-4,27$ & $-0,75$ & 175,88 & $-3,63$ & $-2,5$ & 246,84 & $-13,65$ & $-9,63$ \\
\hline Apr-09 & 227,26 & $-3,36$ & $-1,38$ & 175,94 & $-3,39$ & $-2,7$ & 249,68 & $-10,79$ & $-10,59$ \\
\hline May-09 & 228,44 & $-2,30$ & $-1,81$ & 175,95 & $-3,26$ & $-2,9$ & 252,60 & $-7,22$ & $-10,91$ \\
\hline Jun-09 & 229,15 & $-1,69$ & $-2,12$ & 175,72 & $-3,31$ & $-3,0$ & 253,95 & $-4,96$ & $-10,85$ \\
\hline Jul-09 & 229,64 & $-1,40$ & $-2,37$ & 175,43 & $-3,28$ & $-3,2$ & 254,29 & $-4,26$ & $-10,69$ \\
\hline Aug-09 & 230,19 & $-0,97$ & $-2,54$ & 175,35 & $-2,95$ & $-3,3$ & 254,18 & $-3,73$ & $-10,43$ \\
\hline
\end{tabular}

\begin{tabular}{|c|c|c|c|c|c|c|c|c|}
\hline \multicolumn{4}{|c|}{ CONSTRUCCIÓN } & \multicolumn{2}{|c|}{ COMERCIO } & \multicolumn{3}{|c|}{ HOTELES } \\
\hline & $\begin{array}{c}\text { IMAE } \\
\text { variación } \\
\text { interanual }\end{array}$ & $\begin{array}{l}\text { Prom. } \\
\text { últimos } \\
12 \text { meses }\end{array}$ & & $\begin{array}{l}\text { IMAE va- } \\
\text { riación in- } \\
\text { teranual }\end{array}$ & $\begin{array}{l}\text { Prom. } \\
\text { últimos } \\
12 \text { meses }\end{array}$ & & $\begin{array}{c}\text { IMAE } \\
\text { variación } \\
\text { interanual }\end{array}$ & $\begin{array}{l}\text { Prom. } \\
\text { últimos } \\
12 \text { meses }\end{array}$ \\
\hline 303,86 & 7,09 & 14,59 & 177,90 & 2,52 & 6,43 & 379,94 & $-0,61$ & 3,66 \\
\hline 302,91 & 6,39 & 13,53 & 177,01 & 1,23 & 5,90 & 376,40 & $-1,94$ & 3,07 \\
\hline 302,27 & 5,79 & 12,48 & 175,95 & $-0,18$ & 5,23 & 372,84 & $-3,38$ & 2,35 \\
\hline 301,82 & 4,92 & 11,48 & 174,96 & $-1,49$ & 4,42 & 369,89 & $-4,49$ & 1,54 \\
\hline 301,07 & 2,74 & 10,33 & 174,10 & $-2,57$ & 3,52 & 367,23 & $-5,34$ & 0,70 \\
\hline 297,21 & $-0,82$ & 8,83 & 173,22 & $-3,53$ & 2,56 & 364,53 & $-6,22$ & $-0,22$ \\
\hline 294,27 & $-3,38$ & 7,08 & 172,35 & $-4,35$ & 1,57 & 362,12 & $-7,01$ & $-1,20$ \\
\hline 295,53 & $-4,14$ & 5,33 & 171,78 & $-4,72$ & 0,58 & 359,82 & $-7,67$ & $-2,21$ \\
\hline 297,60 & $-4,32$ & 3,75 & 171,51 & $-4,70$ & $-0,34$ & 357,33 & $-8,23$ & $-3,24$ \\
\hline 297,26 & $-4,57$ & 2,37 & 171,27 & $-4,61$ & $-1,18$ & 354,92 & $-8,57$ & $-4,23$ \\
\hline 295,62 & $-4,73$ & 1,13 & 170,94 & $-4,57$ & $-1,94$ & 352,96 & $-8,61$ & $-5,12$ \\
\hline 294,11 & $-4,30$ & 0,06 & 170,60 & $-4,47$ & $-2,62$ & 351,62 & $-8,26$ & $-5,86$ \\
\hline 293,38 & $-3,45$ & $-0,82$ & 170,25 & $-4,30$ & $-3,19$ & 350,62 & $-7,72$ & $-6,45$ \\
\hline
\end{tabular}

